山्山FFANÇAISE

$\supset \mathrm{DE}$

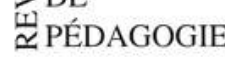

\section{Revue française de pédagogie}

Recherches en éducation

159 | avril-juin 2007

Politique et rhétoriques de l'« École juste » avant la Cinquième République

\title{
L'« égalité dans la diversité » : un modèle de justice à la préhistoire de l'école unique (1898-1914)
}

"Equality within diversity": a model of justice in the prehistoric time of the $k$ to-12 school system

La "igualdad en la diversidad" : un modelo de justicia en la prehistoria de la escuela única

"Gleichheit in der Verschiedenheit“: ein Gerchtigkeitsbeispiel in den Anfängen der Einzigen Schule

\section{Frédéric Mole}

\section{OpenEdition}

Journals

Édition électronique

URL : http://journals.openedition.org/rfp/665

DOI : $10.4000 /$ rfp. 665

ISSN : 2105-2913

Éditeur

ENS Éditions

Édition imprimée

Date de publication : 1 avril 2007

Pagination : 23-34

ISBN : 978-2-7342-1090-0

ISSN : 0556-7807

\section{Référence électronique}

Frédéric Mole, «L'« égalité dans la diversité » : un modèle de justice à la préhistoire de l'école unique (1898-1914) », Revue française de pédagogie [En ligne], 159 | avril-juin 2007, mis en ligne le 01 avril 2011, consulté le 20 avril 2019. URL : http://journals.openedition.org/rfp/665; DOI : 10.4000/rfp.665 


\section{$L^{\prime}$ «égalité dans la diversité »: un modèle de justice à la préhistoire de l'école unique (1898-1914)}

Frédéric Mole

Dans les années 1900, l'organisation de l'enseignement en ordres séparés fait l'objet de critiques répétées. Les projets d'unification visant à étendre la détection des aptitudes et à fonder une nouvelle égalité scolaire sont portés par les radicaux-socialistes, au premier rang desquels Ferdinand Buisson. Pour se développer, ce mouvement réformateur doit prendre en compte les postures politiques qui voient dans l'unification divers périls. Le défi consiste à imaginer une certaine forme de mise à égalité des élèves dans un contexte de tensions politiques où la République est mise à l'épreuve du conservatisme et du socialisme. À travers des stratégies argumentatives composant avec les idéologies en présence, les réformateurs cherchent à élaborer et à promouvoir un système d'enseignement démocratique conçu sous le régime de l'égalité dans la diversité : un enseignement secondaire ouvert à tous, aux parcours très diversifiés et respectant les aptitudes de chacun. La version de l'unification scolaire ainsi esquissée annonce un nouveau pari démocratique tant pour l'école que pour la société. Elle inaugure le processus de constitution du référentiel de l'école unique et contient peut-être aussi en germe ses crises ultérieures.

Descripteurs (TEE) : Buisson (Ferdinand), démocratie, égalité de chances, France, politique d'éducation, socialisme.

\begin{abstract}
$\grave{A}^{\prime \prime m i n g}$ a fin $d u X^{e} X^{e}$ siècle, l'organisation de l'enseignement en ordres hiérarchisés, qui reconduit massivement les inégalités par la répartition des élèves dans des réseaux très cloisonnés, semble dessiner un univers indépassable. Les républicains conservateurs concèdent que l'histoire scolaire des individus résulte d'une prédestination sociale, mais ils estiment celle-ci inévitable et légitime, la stabilité d'une reproduction sociale leur paraissant préférable aux périls d'un système ouvert. Les divers courants poli-
\end{abstract}

tiques visant l'égalité sociale (socialistes ou libertaires) ne s'accordent pas, quant à eux, à revendiquer la généralisation de l'accès à l'enseignement secondaire des meilleurs élèves issus de la "classe des producteurs » et s'attachent à une défense - même critique - de l'école primaire comme école du peuple. Deux courants politiques opposés concourent donc à établir l'inévitable coïncidence générale entre origine et destination sociales dans l'institution scolaire. Dans les années 1900, ce modèle cloisonné 
commence à se fissurer sous l'impulsion réformatrice radicale-socialiste. Une unification des enseignements primaire et secondaire est réclamée. Elle devrait prendre la forme d'une ouverture de tous les parcours à tous les élèves selon les aptitudes de chacun. Le modèle esquissé tendrait à briser le carcan des ordres d'enseignement par un enseignement secondaire recomposé en une pluralité d'établissements et de filières traitant la diversité des élèves par une sélection équitable.

Comment ce modèle a-t-il été élaboré ? De l'enquête parlementaire sur l'enseignement secondaire de 1898 (cf. Ribot, 1898) à la première guerre mondiale, cette poussée vers l'unification s'exprime notamment dans la presse, au sein de la commission parlementaire de l'enseignement, dans des congrès, qualifiés par Ferdinand Buisson de "laboratoires populaires où la pensée démocratique jaillit »(Buisson, 1906b), et surtout à travers plusieurs propositions de lois. Comment rendre compatibles, dans l'organisation de l'enseignement, le principe d'égalité qui sous-tend idéologiquement le projet républicain et le principe de diversité qui doit organiser la reconnaissance des aptitudes individuelles et leur distribution dans les parcours scolaires et les carrières professionnelles ? Comment surmonter le double obstacle, conservateur et révolutionnaire, à l'unification et trouver la synthèse entre nécessaire sélection et exigence démocratique ? Tels sont les problèmes que les réformateurs tentent de résoudre. Une stratégie rhétorique se développe pour composer avec les forces sociales en présence, tenter de ressaisir les arguments adverses, surmonter dialectiquement la tension politique entravant la marche à l'unification, rendre recevable ce renversement de perspective.

Alors que conservateurs et révolutionnaires se rejoignent, paradoxalement, dans l'idée que l'école ne peut que refléter toutes les hiérarchies (scolaires, professionnelles et sociales), les radicaux veulent croire qu'elle pourrait au contraire contribuer à modifier la répartition sociale. Dépasser, d'une part, l'élitisme archaïque de la distribution héréditaire des carrières au profit d'une autre logique élitaire adaptée aux exigences d'efficacité de l'économie moderne ; recycler, d'autre part, pour l'adapter au monde présent, le projet socialiste d'« éducation intégrale » jusqu'alors inscrit dans l'utopique perspective égalitariste d'une société sans classes ; telle est la double ambition des radicaux-socialistes. II s'agit donc d'examiner - en étant attentif à repérer, dans ce processus, les continuités subsistant sous l'apparente rupture - comment les réformateurs traitent les positions adverses, se saisissent de leurs thématiques, déplacent et détournent leurs arguments, et recomposent enfin, autour du thème de l'«égalité dans la diversité », un nouveau modèle d'école juste. Ce travail cherche à préciser le rôle tenu, avant 1914, par Buisson et les radicaux-socialistes dans l'élaboration de la conception de l'égalité scolaire du $x^{e}$ siècle - même si aucune loi essentielle n'est alors adoptée - et d'indiquer en quoi la constitution du référentiel de l'école unique s'en trouve durablement marquée, puisque l'idée d'une égalité dans la diversité se lit encore dans le plan Langevin-Wallon: "Le premier principe, celui qui par sa valeur propre et l'ampleur de ses conséquences domine tous les autres est le principe de justice. Il offre deux aspects non point opposés mais complémentaires : l'égalité et la diversité ». Tous les débats sur ce que pourrait être une école démocratique s'articulent alors autour de quelques questions entrelacées dont il faut suivre les principaux déplacements d'une posture à l'autre et identifier le mode de traitement dans les stratégies réformatrices: l'accomplissement de l'individu dans un genre de vie, la possibilité de donner une chance égale à chacun, les risques sociaux du déclassement, l'équitable distribution des positions sociales.

\section{CRISE DES ORDRES D'ENSEIGNEMENT ET RHÉTORIQUE RÉACTIONNAIRE}

L'idée d'une éducation scolaire qui réconcilierait la pluralité des formes de cultures et abolirait les clivages entre ordres d'enseignement est déjà présente, à la fin $d u X X^{e}$ siècle, dans les analyses socialistes révolutionnaires à travers la notion d'"éducation intégrale ». Un syntagme constamment utilisé dans les congrès socialistes à l'appui d'un projet à la fois scolaire, économique et politique de suppression de toutes les formes de hiérarchies culturelles, professionnelles ou sociales. Selon cette optique politique, l'institution scolaire ne saurait être réorganisée de façon égalitaire sans une transformation profonde de la société. Dès 1869, dans son article fondateur sur l'enseignement intégral, reprenant ses contributions sur le même thème au sein de la I $^{\text {re }}$ Internationale, Paul Robin avait expliqué que l'éducation intégrale ne s'inscrivait pas dans la perspective d'une ascension sociale des meilleurs : le travailleur manuel « comprendra, en effet, qu'il ne suffit pas qu'il s'efforce de quitter sa position pour aller chercher ailleurs le bonheur auquel il sent qu'il a droit, mais que, comme sa fonction doit être remplie, il faut que l'organisation sociale 
se modifie de telle sorte qu'il puisse être heureux là où il est. En un mot, il ne cherchera pas à jouir d'un nouveau privilège, mais à obtenir pour lui et les autres le simple dû selon la justice » (Robin, 1869, p. 272).

La question scolaire étant subordonnée à la perspective d'une émancipation sociale de la classe des producteurs, toute réforme est suspectée de viser à fournir l'instrument d'une entreprise de détection des aptitudes les plus utilisables au regard des intérêts de la «classe dominante». Parce qu'ils redoutent, dans un processus d'unification, l'effet pervers du «drainage » des «forces vives » du prolétariat récupérées au profit de la "caste dirigeante » (Guesde, 1899, p. 309), parce qu'ils refusent de voir favorisée l'ascension sociale de quelques uns et la masse sacrifiée, les courants révolutionnaires s'accommodent d'un dualisme scolaire qui, sans être juste, ne leur paraît pas inopportun du point de vue de leur stratégie de défense critique de l'école primaire comme école du peuple. Si les socialistes modérés sont parfois plus nuancés sur la question, Jaurès lui-même, commentant les résolutions du Congrès de la Ligue de l'enseignement de 1906, s'inquiète, à la une de L'Humanité, de la « désharmonie » qui résulterait de ce que l'on commence par «supprimer les classes sociales dans l'organisation scolaire sans les supprimer dans la société elle-même » (Jaurès, 1906) (1).

Bien que les principaux promoteurs socialistes ou anarchistes de l'idée d'éducation intégrale ne s'engagent pas dans le mouvement pour l'unification, les analyses républicaines conservatrices désignent - et dénoncent - cependant cette doctrine éducative comme la principale source d'inspiration de l'idéologie unificatrice. Apparent paradoxe seulement, si l'on est attentif au fait que la rhétorique conservatrice consiste précisément à imputer la critique des ordres d'enseignement au développement de la pensée socialiste en général. Lorsqu'en 1898, Alexis Bertrand, professeur de philosophie à l'université de Lyon, publie L'enseignement intégral, il n'échappe pas aux commentateurs que cet ouvrage d'orientation positiviste, examinant les conditions d'une recomposition et d'une ouverture de l'enseignement secondaire, tend à donner un crédit universitaire à une notion jusqu'alors principalement mobilisée au sein des mouvances révolutionnaires et ouvre ainsi la voie à de nouvelles ambitions réformatrices. $\mathrm{Si}$, comme le remarque Daniel Hameline, A. Bertrand luimême se montre «plus que discret sur cette filiation ouvriériste » (Hameline, 2004), Gabriel Compayré et Alfred Fouillée, dans leurs longs commentaires de l'ouvrage, saisissent au contraire l'occasion de faire le procès de l'idée socialiste d'éducation intégrale et s'emploient à reformuler rigoureusement la doctrine des ordres d'enseignement.

Retenons, parmi les recensions de l'ouvrage, celle, emblématique, d'Alfred Fouillée. Face à la " chimérique " tentation socialiste de "supprimer ou fondre les classes dans l'universelle harmonie ", le sociologue, dont le conservatisme scolaire s'inscrit paradoxalement dans le cadre d'une pensée pourtant socialement progressiste, cherche à montrer la légitimité des inégalités constatées dans les cheminements scolaires et à justifier la reproduction par l'école de la répartition professionnelle et sociale d'une génération à l'autre. La diversité des aptitudes correspondrait, selon lui, à une "classification naturelle et spontanée » qui doit être respectée «au nom du droit de chacun comme au nom de l'intérêt social ". A. Fouillée invoque même un droit de l'individu à être éduqué conformément à ses aptitudes, c'est-à-dire ni en deçà, ni au-delà. La légitimité d'une telle classification serait liée à une «loi d'hérédité et d'accommodation au milieu " que la répartition des aptitudes entre travail manuel et travail intellectuel lui paraît illustrer. Une telle « loi », qui semble faire écho à la théorie de l'hérédité des caractères acquis, justifie les inégalités scolaires en amalgamant facteurs naturels et facteurs sociaux dans un ordre unique et nécessaire et atteste un biologisme social d'orientation eugéniste : les « enfants de laboureurs robustes [naissent] généralement robustes et particulièrement propres à travailler la terre, plutôt qu'à faire du calcul infinitésimal ou de la philosophie ", tandis qu'on voit les « classes intellectuelles [procréer] en plus grande abondance moyenne des enfants moins robustes, mais à cerveau déjà développé et malléable, tout prêt à l'exercice ». Les exceptions observées ne sauraient remettre en question, estime $A$. Fouillée, l'existence de «classes manuelles dont l'auteur est l'hérédité » (Fouillée, 1898).

Cette conception ne heurte d'ailleurs guère l'air du temps républicain : dès lors que l'on peut poser avec Octave Gréard que « la valeur d'un homme consiste non à ressembler tant mal que bien à tous les autres, mais à réaliser la perfection de sa nature » (Gréard, 1889), toutes les hiérarchies intellectuelles, professionnelles ou sociales peuvent être admises comme autant de différences justifiées a priori. On remarque au passage que l'argument de la diversité des formes d'intelligence, de nos jours plus souvent utilisé en faveur de tentatives de redéfinitions démocratiques des programmes, était alors invoqué à l'appui des cloisonnements scolaires. De la même façon, lorsqu'il 
lui est demandé, dans le cadre de l'enquête parlementaire sur l'enseignement secondaire de 1899, s'il serait opportun, dans un esprit démocratique, de supprimer les petites classes élémentaires des lycées et de faire obligation à tous les élèves de recevoir l'instruction au sein des écoles primaires, A. Fouillée répond : «Ne mêlons pas à tort et à travers les diverses couches sociales et les divers milieux sociaux. Tout doit être ouvert, rien ne doit être confondu. II y a des classifications naturelles et spontanées qu'il faut respecter»(Ribot, 1899, p. 274). La position conservatrice peut donc s'énoncer de la façon suivante. Seuls les ordres d'enseignement, classant les aptitudes des individus les plus prévisibles au regard de leurs appartenances sociales, peuvent assurer une distribution viable, et donc juste, des parcours et garantir une sélection des élites qui se trouverait au contraire mise en péril par l'unification : " Le triage est le procédé universel de la nature, et il est aussi celui de la morale. Les brebis galeuses ne tardent jamais à infester le troupeau. En voulant rendre toute l'éducation trop uniforme, on risque de rabaisser les âmes d'élite aux âmes vulgaires. La vraie démocratie, même socialiste, devrait donc respecter partout la hiérarchie et la favoriser, au lieu de poursuivre le nivellement de tout et de tous. Jusqu'à ce que I'humanité ait atteint un stade encore bien loin de nous, les trois degrés de l'instruction, sans être fermés l'un à l'autre, devront demeurer distincts, comme répondant à des "couches sociales" diverses, et aussi à des couches morales qu'il est dangereux et injuste de mêler » (Fouillée, 1898, p. 582).

Renoncer aux ordres d'enseignement, ce serait renoncer à classer les individus et annoncer des troubles sociaux. Fouillée explique que, sous un tel régime d'«inclassement universel » - syntagme formé pour l'occasion -, le principe de l'égalité d'instruction conduirait à admettre un principe d'équivalence entre les individus. Or, « en voulant rendre chacun propre à tout, on rendrait chacun impropre à tout » (Ibid., p. 581). L'unification ne consisterait donc qu'en une uniformisation. Quant à l'égalité scolaire, elle serait illusoire parce que sans issue professionnelle. Le " grand péril » d'une « instruction encyclopédique d'État pour tous » résiderait dans «l'éveil d'ambitions impossibles à satisfaire » et le «manque d'adaptation des esprits à leur future condition sociale». Telles sont les menaces de « démoralisation » et de “ déséquilibration » (Fouillée, 1898) que ferait porter le «socialisme pédagogique », selon une dénomination utilisée par G. Compayré (1898) et A. Fouillée (1898).
S'exprime donc ici une tendance profonde du conservatisme républicain, tolérant les « exceptions consolantes» rendues possibles par le système des bourses - des exceptions, remarque Buisson, "propres à faire oublier l'injustice foncière qui reste la règle générale » (Buisson et al., 1910, p. 7) - mais non l'ouverture de l'enseignement secondaire au plus grand nombre ni, moins encore, un même enseignement élémentaire pour tous (Fouillée, 1898, p. 576). Sur le système des bourses, il faut signaler une certaine convergence de vues entre les socialistes révolutionnaires, qui y voient un de ces « palliatifs bourgeois contre l'insuccès de l'école » (Parti ouvrier socialiste révolutionnaire, 1897, p. 71), et Ferdinand Buisson qui proposera plus tard son remplacement par un autre régime fondé non sur la « faveur » mais sur un «droit à une aide normale de la société, que tous les jeunes gens, filles et garçons, pourront réclamer » (Buisson, 1933 [1914]).

\section{DROIT DE L'INDIVIDU ET PÉRIL DU DÉCLASSEMENT}

Face à des postures antagonistes qui ont en commun de refuser de voir dans le dépassement du dualisme scolaire la condition d'un progrès économique ou d'une espérance démocratique, les radicaux envisagent une réforme scolaire qu'ils estiment susceptible de précéder, préparer et accompagner l'émergence d'une société nouvelle. L'individu devrait y trouver la fonction qui lui convient au regard de ses aptitudes et de l'intérêt collectif et, par là, les conditions de son accomplissement personnel. Jusqu'alors arrimé à une appartenance, l'individu se voyait prescrire une destination. On avait pu considérer que le genre de vie professionnelle convenant à un individu lui était prescrit par un héritage biologique et social, ou bien qu'il était lié à l'appartenance à une classe sociale qu'on estimait investie d'une mission historique émancipatrice. Ce qui revenait toujours à craindre, d'un côté comme de l'autre, que les individus, s'écartant de leurs appartenances initiales en se dessinant des destinations singulières, ne perturbent les rapports de force en présence. Dans tous les cas, sortir de sa classe, c'était ne plus être à sa place et c'était mettre en péril des cadres normatifs qui, bien qu'opposés au plan politique, confortaient le dualisme scolaire.

Les radicaux prétendent dépasser ces deux modèles tout en conservant certains de leurs arguments majeurs. Le débat est lisible notamment à travers la 
thématique du "déclassement », catégorie rhétorique commune à toutes les postures politiques du moment. Selon la définition qu'en donne Buisson, les déclassés sont " ceux qui, ne voulant plus rester dans leur classe, n'ont pas pu entrer dans l'autre " (Buisson et al., 1910, p. 8). La notion renvoie le plus souvent à la contradiction sociale vécue par ces enfants du peuple chez qui l'accession à une culture scolaire plus élevée que celle que ne laissait prévoir leur appartenance sociale suscite l'espérance, le plus souvent déçue, d'une nouvelle position dépassant cette origine. Mais le thème est réversible : le conservatisme craint, par trop de démocratie scolaire, un déclassement par le bas des anciennes élites. Dans tous les cas, on l'a vu, le phénomène inquiète. L'extension démesurée de la proportion des déclassés transformerait à coup sûr l'ambition personnelle en une attente - voire une revendication - collective qui aboutirait à la mise en péril de la répartition et de la paix sociales. De l'autre point de vue, socialiste révolutionnaire, le déclassement est vu comme l'effet pervers d'une espérance démocratique ramenée au seul désir d'une ascension sociale pour quelque uns et il traduit la situation de ceux qui, issus de la " classe dominée ", en oublient les souffrances et la trahissent. Mise en péril et effet pervers - au sens d'Albert Hirschmann (1991) - jouent ici comme deux rhétoriques inversées mais convergentes.

Face à cette collusion entre un rigorisme élitaire et un rigorisme égalitaire, obstacle au mouvement pour le « droit des enfants devant l'instruction », les réformateurs annoncent vouloir en finir avec «ce préjugé ridicule et stupide du déclassé » (Briand, 1906) qui pérennise une conception fixiste de la diversité des parcours, calquée sur la répartition sociale. Mais la prudence s'impose et Buisson, dans son discours au $X X V I^{e}$ congrès de la Ligue de l'enseignement, à Angers, tente de donner des gages aux deux parties : «Nous ne voulons pas plus de déclassés dans la classe ouvrière que de déclassés dans la classe bourgeoise »(Buisson, 1906c, p. 444). Pour persuader les différents adversaires de l'unification, il faut élaborer une argumentation qui à la fois emprunte aux objections et y réponde. Buisson assure, d'une part, que la réforme ne vise ni le « nivellement des esprits" ni celui des fonctions et qu'elle n'implique nullement d'«abolir la propriété individuelle» ou d'“établir d'un coup l'égalité des professions et des salaires » (Buisson et al., 1910, p. 13). Mais il annonce, d'autre part, que si l'unité d'éducation « ne supprimera pas instantanément la lutte des classes », elle «tendra rapidement, ce qui vaut mieux, à supprimer les classes ». Et pour récuser plus particulièrement la thèse socialiste d'un effet pervers de la réforme, il promet que celle-ci «n'aura pas pour effet de faire émigrer quelques individus de la classe ouvrière pour leur faire prendre la mentalité de la classe privilégiée où ils entrent », mais qu'elle consistera au contraire à " élever la classe ouvrière à la conscience d'un droit égal à celui de la classe bourgeoise » (Ibid., p. 16).

En référant le projet d'unification scolaire aux principes socialistes, Buisson cherche à l'inscrire dans I'horizon politique de l'égalité sociale. Tout se passe comme si, en réponse à l'utopie éducative socialiste révolutionnaire, Buisson était amené à élaborer une sorte de contre-utopie réformatrice : celle consistant à rechercher les conditions de possibilité d'une mise à égalité scolaire des individus au sein d'une société demeurant inégalitaire. Une visée politique qui ne suppose plus de subordonner la réforme démocratique de l'école à une hypothétique révolution sociale.

\section{UNE PREMIÈRE ÉGALITÉ DE “CHANCE "? À CHACUN LA PLACE QUE LUI ASSIGNENT SES APTITUDES}

Comment proclamer l'égalité des enfants devant l'instruction et garantir dans le même temps le libre déploiement des inégalités d'aptitudes ? Comment ressaisir l'idée d'éducation intégrale, afin de tenter d'inscrire la réforme dans le sillage des revendications socialistes, tout en assurant que les hiérarchies scolaires seront respectées? Les projets les plus significatifs élaborés par les républicains de gauche, en particulier les propositions de loi de Maximilien Carnaud en 1909 et de F. Buisson en 1910 (Briand \& Chapoulie, 1992), cherchent à prendre en compte sur le plan argumentatif les résistances à l'idée d'unification en élaborant un nouveau modèle de la diversité scolaire fondé sur une pluralité de parcours potentiellement ouverts à tous au sein d'un enseignement secondaire présenté comme unifié bien que sans tronc commun. Face à un système cloisonné considéré comme doublement archaïque - injuste et inacceptable au regard des attentes sociales, inadapté et inefficace du point de vue d'une détection des aptitudes - le contremodèle que Buisson propose sous le nom de "système démocratique d'éducation nationale " devrait ainsi être conçu "sous le régime de l'égalité dans la diversité » (Buisson et al., 1910, p. 16).

Afin de concilier égalité et sélection, les radicaux procèdent donc à un travail de réappropriation de la notion d'« éducation intégrale " qu'ils réinterprètent 
selon une logique libérale. Originellement lié à l'affirmation d'un droit de chaque individu au développement complet de toutes ses aptitudes (et pas seulement des meilleures) dans le cadre d'une critique de la division du travail, le concept renvoie désormais à un dispositif systématique de détection des aptitudes les plus spécifiques de chacun et de distribution de ces aptitudes dans une pluralité de parcours de formation. Par ce renversement, l'éducation intégrale ne se définit donc plus comme la condition d'un accès de chaque individu à la variété des formes de cultures et elle se trouve paradoxalement réinscrite dans une division du travail à laquelle on cherche à donner une nouvelle légitimité. Certes, en ancrant les aptitudes de l'individu dans sa singularité propre, on cherche en un certain sens à l'affranchir de la superstition de l'origine sociale, à le rendre à lui-même et à lui rendre justice ; et on fait un devoir à la nation de "veiller à ce que pas un de ses enfants ne soit privé des moyens d'accomplir intégralement et normalement toute l'évolution dont la nature l'a rendu capable " (Buisson et al., 1910). Mais c'est seulement en le développant "conformément à sa nature propre" qu'on entend faire de chaque individu "un être complet " (Thalamas, 1906, p. 6). L'exigence de justice ici affirmée ne repose donc plus sur la critique des spécialisations scolaires, culturelles ou professionnelles: elle tiendrait désormais à ce que chacun devrait se voir assigner par l'institution la place correspondant à ses aptitudes les meilleures, telles qu'elles auraient été reconnues objectivement au moyen de dispositifs " rationnels " et " scientifiques " de sélection (Carnaud, 1909). Le caractère quelque peu paradoxal de cette réinterprétation de la notion d'éducation intégrale est repérable dans la courte formule figurant dans le programme du Parti radical adopté en 1907 (art. 10) : "Tous les enfants du peuple ont droit à l'éducation intégrale suivant leur aptitudes » (Buisson, 1908b, p. 194). S'il est donc un " droit de tous les enfants [...] à une éducation intégrale ", Buisson précise que cette dernière expression désigne la diversité des «types d'enseignement et d'éducation correspondant à tous les besoins de la société » (Buisson et al., 1910, p. 2 ; souligné dans le texte). C'est à l'échelle de la société, et non plus à l'échelle de l'individu, que l'éducation intégrale serait considérée comme réalisée. On s'est quelque peu éloigné de la revendication d'un "droit ", pour tout homme, de "développer, le plus complètement possible, toutes ses facultés physiques et intellectuelles " (Robin, 1869, p. 271 ; je souligne).

L'idée centrale qui fonde la réforme est donc celle d'une distribution « en toute justice » de l'instruction.
Ce qui signifie que celle-ci devra être "proportionnée aux facultés de chacun "(Carnaud, 1909, p. 7), facultés qu'il convient dès lors d'identifier rigoureusement. Dans le cadre de cette justice distributive, l'ambition démocratique de la réforme est à la fois affirmée et bornée. Les réformateurs sont par exemple convaincus de la nécessité d'éviter l'afflux massif d'élèves candidats à de longues études : "II ne faudrait pas croire que cette égalité consisterait à obliger les enfants à passer nécessairement et par voie de concours de l'école au lycée puis à la Faculté. Ce serait là une vue étroite et très dangereuse. Les carrières libérales ou scientifiques tiennent déjà une place excessive dans notre société " (Thalamas 1906). La réforme repose donc à la fois sur l'affirmation d'un droit de l'individu et sur la nécessité de circonscrire rigoureusement ses meilleures aptitudes. Ce recours à une version révisée du concept d'éducation intégrale présente un double avantage stratégique : tout en référant la réforme à une revendication égalitaire, à une demande de justice sociale, on donne par ailleurs des gages aux défenseurs de la hiérarchie en promettant que cette unification n'entraînera aucune uniformisation susceptible d'entraver le recrutement des élites (2).

La proposition de loi de 1909, présentée par M. Carnaud au nom de la commission de l'Enseignement et des Beaux Arts de l'Assemblée Nationale, témoigne d'un souci particulier de prévenir les risques d'uniformisation que pourrait comporter la mise en œuvre d'un régime scolaire démocratique. Évoquant les travaux d'Édouard Toulouse, M. Carnaud explique qu'il ne saurait jamais y avoir égalité d'aptitudes chez les êtres humains et formule une mise en garde qui rappelle les arguments conservateurs relatifs au péril démocratique : "L'ambition de vouloir être plus que ne le comportent ses possibilités " est une "source de tourments pour l'individu » et une « cause de grave désordre dans le social ». Suivent de très longs développements sur les dispositifs propres à garantir une "sélection rationnelle des aptitudes " susceptible de prévenir de tels désordres. Le projet ne va cependant pas jusqu'à chercher la rationalité de cette sélection dans un recours à des tests psychotechniques. Cette rationalité ne doit pas non plus être attendue des examens et concours, regardés avec suspicion parce que trop aléatoires. On préfère confier la responsabilité de la sélection aux maîtres eux-mêmes dont le regard est estimé bien "plus sérieux » et qu'on invitera à s'instruire dans "l'art de rechercher et de découvrir les différences corporelles, sensorielles, intellectuelles, psychiques... de leurs élèves ". À tous les échelons, l'institution serait impliquée dans cette 
tâche. Les inspecteurs des divers enseignements collecteraient chaque année, à l'usage de commissions régionales, des "fiches signalétiques spéciales» indiquant pour tous les élèves leurs "qualités ", leurs "prédispositions les plus remarquables" ainsi que la « désignation soit de l'école d'un degré immédiatement supérieur, soit du cours professionnels, suivant le cas, qui conviendrait le mieux à la culture de leurs facultés". Une " commission supérieure interministérielle de sélection des aptitudes des élèves et de coordination des enseignements " serait en outre chargée de "rétablir l'unité » entre tous les enseignements. On peut considérer qu'à certains égards ce texte annonce et préfigure le développement conjoint, après la guerre, des problématiques de l'école unique et de l'orientation.

\section{LA « DIVERSITÉ PÉDAGOGIQUE DANS L'ÉGALITÉ SOCIALE "?}

Ferdinand Buisson salue la proposition de M. Carnaud mais s'en démarque en laissant entendre que le dispositif de sélection envisagé pourrait comporter le risque de « mettre à part les sujets d'élite ». Évoquant le rapport qu'il avait rédigé à l'occasion du $9^{e}$ congrès du Parti radical, à Nantes en octobre 1909, Buisson rappelle son souci d'éviter de « faire évader de la "classe ouvrière" les sujets les plus méritants et les plus distingués » et il formule ce qui lui paraît être une condition essentielle de la réforme envisagée : «La nation considèrerait comme équivalentes toutes les formes de l'activité humaine : manuelle ou intellectuelle, agricole ou commerciale, industrielle ou artistique, scientifique ou littéraire » (Buisson, 1909).

En 1910, Buisson dépose une proposition de loi qui reprend et complète le rapport adopté à l'unanimité par le $9^{e}$ congrès du Parti radical. L'argumentation est centrée sur la nécessité de concilier deux principes fondateurs de la réforme. D'une part, donner à chaque individu une chance égale de développement; d'autre part, chercher les conditions d'une rentabilisation des aptitudes. Le texte affirme donc d'abord que toute institution qui opposerait " des obstacles [...] au droit naturel de chaque enfant, à ses chances de développement normal ", devrait être considérée comme «injuste » et « antisociale». Mais pour ajouter aussitôt que la nation devant « tirer parti de toutes les ressources que la nature lui offre ", il s'agit bien d'" utiliser tous les individus selon leurs aptitudes et de porter ces aptitudes par l'éducation à leur maximum de rendement ». Pour poursuivre ces deux buts à la fois, il convient de concilier généralité et spécialisation. L'enseignement secondaire devrait donc donner à tous les élèves au-delà du primaire une éducation que Buisson tient à qualifier de "générale et commune » mais qui serait distribuée à des élèves répartis «par groupes déjà subdivisés en raison de leur futures professions éventuelles » (Buisson et al., 1910, p. 13). On voit donc qu'aucun tronc commun ne serait envisagé à ce niveau d'enseignement, qui devrait se décliner dans des établissements très diversifiés : «À la différence du premier cycle, [le deuxième cycle] ne comporte plus un seul et même genre d'établissements. Et c'est à ce moment que va commencer à se déployer la diversité pédagogique dans l'égalité sociale. La nouveauté sera précisément de mettre sur le même pied, au regard de la société, de traiter exactement avec la même faveur, avec les mêmes soins et comme ayant un prix égal, les modes divers d'éducation qui aujourd'hui se différencient comme étant de valeur différente et se classent par étages superposés. En démocratie véritable seront classés sous le titre d'écoles secondaires - diverses d'objets, égales en valeurs sociales - des établissements scolaires beaucoup plus variés qu'aujourd'hui, organisés en vue de donner à l'adolescence des enseignements encore généraux et préparatoires, mais déjà orientés et dirigés en vue des adaptations ultérieures. Égalité d'instruction ne signifie pas identité d'enseignement. » (Buisson et al., 1910, p. 11)

Un double pari fonde l'entreprise par laquelle Buisson tend à impulser une nouvelle équité scolaire. D'une part, l'accessibilité en droit de toutes les filières à tous les élèves doit pouvoir suspendre - ou même renverser - la prédestination scolaire (les seules limites étant celles des aptitudes avérées). D'autre part, il est possible de décréter l'égale dignité des différentes filières d'enseignement (« comme ayant un prix égal ») et ce n'est qu'à cette condition que la diversité des parcours réclamée par la logique élitaire ne serait pas contraire au principe d'égalité. Si Buisson retient du capitalisme la nécessité de rentabiliser les individus et s'il juge utopique la stratégie révolutionnaire faisant de l'égalité sociale le préalable à l'égalité scolaire, il retient cependant des analyses socialistes l'idée qu'une répartition professionnelle ne peut être considérée comme démocratique qu'à la condition d'assurer l'égale dignité sociale de toutes les carrières (3). On peut penser qu'en insistant sur une lecture de la société en termes de classes, Buisson cherche à rallier le camp socialiste au projet d'unification. En somme, demande est faite aux socialistes de prendre conscience à leur tour des effets pervers 
de leur politique et d'adopter «le principe de l'égalité des enfants comme acheminement à l'égalité des hommes. " Buisson se fait insistant : "Comme il y a deux classes d'hommes, [...] il y a deux classes d'enfants [...] Toute la question est de savoir si nous voulons, oui ou non, changer cela, interrompre des traditions séculaires, inaugurer un régime d'éducation égalitaire, qui ne sera pas le dernier mot de la révolution sociale, mais qui en pourrait être le premier. Si nous répondons: non [...], nous aurons d'avance renoncé à organiser sur des bases nouvelles la France de demain. Nous aurons donné des gages au conservatisme social. 》 (4)

On lit sans doute ici un des moments inauguraux de l'orientation des politiques scolaires réformatrices qui marqueront le $x x^{e}$ siècle et dont on peut dire qu'elles ne se fondent et ne se légitiment, dans la conscience collective, que d'être porteuses d'une certaine espérance d'égalité sociale. On voit alors Ferdinand Buisson chercher à restaurer - ou plutôt refonder - une certaine croyance politique en l'école républicaine. Cette proposition de loi constitue en effet une étape majeure dans l'impulsion qu'il donne au mouvement radical-socialiste en faveur d'une certaine conception de l'unification scolaire. En 1908, dans le rapport sur "Le complément et l'achèvement de l'œuvre scolaire de la Troisième République » présenté au nom de la commission de l'enseignement au $8^{\mathrm{e}}$ congrès du Parti radical et radical-socialiste (Dijon), Buisson avait traité du " redoutable programme d'assurer intégralement l'instruction populaire ». Constatant l'importance persistante de l'illettrisme en France par comparaison avec les autres nations, il avait reconnu n'avoir plus le courage d'« exalter la beauté de nos lois " quand tout conduisait au contraire à déplorer un état d'“arriération » et d'« infériorité " de «l'instruction populaire française» (Buisson, $1908 b)$ en particulier au regard de l'Allemagne et de la Suisse qui avaient su déjà porter l'obligation scolaire à l'âge de quatorze ans. À plusieurs reprises, durant cette période, Buisson prend ses distances à l'égard de l'œuvre scolaire accomplie depuis près de trois décennies et dénonce, parfois avec vigueur, la prégnance des inégalités sociales dans l'école. Ainsi, en 1909, à Bourg-en-Bresse, à l'occasion de la fête annuelle de l'Amicale primaire de l'Ain, l'ancien directeur de l'Enseignement primaire (de 1879 à 1896) prononce une conférence sur "Le rôle social de l'instituteur » devant six cent instituteurs du département réunis en assemblée générale : «Pourriez-vous soutenir que l'enfant qui naît dans une famille pauvre est mis en possession des mêmes droits, qu'il jouit des mêmes chances dans la vie, qu'il reçoit de la société les mêmes soins que l'enfant d'une famille riche de la bourgeoisie? Vous êtes trop honnêtes pour l'affirmer en classe, pour faire croire aux élèves qu'en effet l'égalité des droits et la plénitude de la liberté sont réalisées dans notre société. Votre conscience vous le défendrait. Voilà précisément ce qui vous rend suspect au conservatisme social [...] Ce dont certains ont peur, et ce qu'ils vous reprochent [...], ce qu'ils craignent, c'est que vous ne fassiez trop de républicains comme vous, de ceux qui veulent que la République se réalise intégralement. Que voulez-vous, mesdames, messieurs ? II n'y a pas moyen que vous ne commettiez pas ce crime-là. » (Buisson, 1910).

De cette conférence, publiée dans le Bulletin trimestriel de l'Amicale primaire et de la Ligue autonome de l'Ain en janvier 1910, ne subsiste qu'un très court extrait, sous ce même titre, dans La Foi laïque, ouvrage paru en 1912. Le passage cité ici ne figure pas dans ce célèbre recueil d'extraits de discours et d'écrits de Buisson qui restitue une vision plus consensuelle de l'école laïque. Certains articles de son Nouveau dictionnaire de pédagogie et d'instruction primaire, paru en 1911, font cependant écho à cette analyse critique de l'inachèvement de l'œuvre scolaire de la Troisième République et témoignent de ce mouvement vers l'unification (Denis, 2006). En 1913, Buisson réitère sa proposition de loi. II définit ensuite «l'école unique et commune » - officialisant en quelque sorte, avant les Compagnons de l'Université nouvelle, l'usage du syntagme -, comme un système d'éducation « unitaire et égalitaire dans sa profonde diversification ". Cette démocratie scolaire, qui doit commencer par accorder à tous les enfants « des chances égales de départ dans la vie " (Buisson, 1930 [1914], p. 95), Buisson la qualifie de « démocratie partielle » et précise qu'il faut la considérer comme une étape « en attendant que le jour vienne de la démocratie intégrale " (Ibid., p. 93). Après la guerre, la formule adoptée par Buisson accentuera encore les tensions internes à cette conception de l'école démocratique: "L'École unique, c'est donc en même temps l'école aussi diversifiée que possible » (Buisson, 1925, p. 541).

\section{RÉFORME SCOLAIRE ET TRANSFORMATIONS SOCIALES}

L'égalité scolaire peut donc précéder l'égalité sociale. Mieux : elle peut la préparer. Le renversement est complet par rapport aux analyses socialistes révo- 
lutionnaires. Buisson veut croire que cette nouvelle forme d'égalité scolaire pourrait se prolonger dans la division du travail, dont il annonce une forme idéalisée : "La société traitera tous ses enfants [...] comme égaux et frères [...] lls continueront de marcher côte à côte dans des conditions de vie professionnelle qui seront différentes mais non inégales. Ils achèveront leur éducation et leur apprentissage, comme ils l'ont commencé, dans un sentiment d'égalité que la dissemblance des carrières ne détruit pas. " (Buisson et al., 1910, p. 13) (5).

Le radical ne renonce pas à l'idéal socialiste, mais l'imagine réalisable dans une société qu'il décrit luimême comme divisée en classes. L'idée d'une promotion scolaire et professionnelle de tous les enfants du peuple, et pas seulement des meilleurs, vient repousser à plus tard - ou rendre inutile ? - l'autre utopie, celle d'une émancipation sociale collective. Avec cet espoir qu'une fois reconnus au sein des élites, ces enfants du peuple se feront les promoteurs des valeurs d'égalité sociale. Lorsque Jaurès s'inquiète, au lendemain du congrès de la Ligue de l'enseignement à Angers, du «terrible déséquilibre » qui pourrait résulter d'un processus d'unification tendant à atténuer "l'inégalité sociale dans l'enseignement » mais qui ne s'accompagnerait pas d'« un effort équivalent pour diminuer cette inégalité dans la vie sociale » (Jaurès, 1906), Buisson répond: «Réalisons entre enfants ce qui n'est pas, dit-on, réalisable entre hommes. Peut-être ces enfants, devenus hommes, ne le trouveront plus si impossible à réaliser. Cet effort social faisant suite à l'effort scolaire, insiste F. Buisson, qui pourra mieux le tenter que ceux-là mêmes qui auront fait à l'école nouvelle l'apprentissage du nouveau régime égalitaire? " (Buisson, 1906a). Par le pari de donner à chacun une même chance de développement, Buisson cherche donc à renverser la logique sociale qui présidait aux destinées de l'enseignement secondaire. On peut penser que ce puissant investissement symbolique dans le pouvoir égalitaire de l'école s'explique en partie par la volonté de contrer le pessimisme socialiste révolutionnaire qui repoussait toute réforme scolaire dans un moment ultérieur à la transformation de la société. La position de Jaurès, quant à elle, semble se situer à un point médian : réforme de la société et réforme scolaire doivent être conduites dans le même temps.

Chez Ferdinand Buisson, la croyance dans la puissance de l'institution scolaire s'accompagne d'un autre renversement : ce n'est plus la société qui est jugée coupable des inégalités visibles dans l'école mais c'est l'école qu'on estime être la cause de la perpétuation des inégalités sociales. Car, pour parier à ce point sur les promesses de transformations sociales que pourrait receler une réforme scolaire, il faut d'abord avoir beaucoup incriminé l'institution qu'on veut profondément réformer. D'où une tendance à exagérer la responsabilité de l'école : “ C'est l'inégalité du régime éducatif qui prépare et qui rend inévitables les inégalités d'ordre économique, politique et social. » (Buisson et al., 1910, p. 16). On observe là un renversement de causalité dont on peut se demander s'il n'a pas eu des effets durables, notamment celui de jeter un voile d'ignorance sur les facteurs culturels, économiques et sociaux des inégalités scolaires.

Certains contemporains alertent déjà Buisson à ce sujet. Dans les courants politiques progressistes, les résistances aux projets d'unification ne sont pas toujours le fait de socialistes ou de syndicalistes révolutionnaires. C'est le philosophe Gabriel Séailles, professeur à la Sorbonne, libre-penseur, ami de Jaurès et de Buisson, qui formule certaines des critiques les plus vives à l'encontre des résolutions adoptées par le $\mathrm{XXVI}^{\mathrm{e}}$ congrès de la Ligue de l'enseignement : "L'égalité devant l'instruction laisse subsister toutes les autres formes de l'inégalité qui est la loi de nos sociétés. Nous retrouvons ici, contre notre attente, sans doute, les effets de ce libéralisme qui met aux prises le fort et le faible, l'homme nu et l'homme armé de toutes pièces, et qui prétend juger, par l'issue de la lutte, du mérite et du courage des combattants [...] La majorité ne peut être qu'une majorité de vaincus ". Les idées de gratuité de l'enseignement secondaire ou de multiplicité des bourses ne sont, aux yeux de G. Séailles, que de vaines mesures qui ne servent qu'à « dissimuler par une fiction mensongère l'inégalité réelle dont on n'a fait que modifier les effets et les formes » (Séailles, 1906).

Ferdinand Buisson, en insistant sur la responsabilité de l'école dans la production des inégalités sociales et sur le pouvoir qu'elle aurait de les atténuer si on la réformait, a sans doute inauguré une nouvelle forme d'espérance qui marquera par la suite l'histoire de l'école unique. La vigueur de cette contre-utopie, tendant à subordonner la réforme de la société à la réforme de son école, n'a-t-elle pas ainsi contribué à dissimuler les processus de maintien des positions sociales, processus dont la sociologie de la reproduction a levé le voile dans les années 1960 ? N'a-t-elle pas également conduit à assimiler espérance démocratique et démocratisation scolaire? 


\section{CONCLUSION}

On assiste, durant cette période, à une mise en crise du modèle de justice qui fondait le dualisme scolaire. Mais on s'aperçoit que ce renversement s'effectue à partir de certains critères de justice dont les divers déplacements se produisent à l'intérieur d'une sorte de matrice fondamentale commune à tous les acteurs. Ceux-ci en effet se déterminent à partir d'un même type de problèmes auxquels ils apportent des réponses opposées, mais aussi parfois étrangement proches dans leurs divergences.

Écartant donc toute rêve d'un enseignement uniforme pour l'ensemble des adolescents, l'unification devait rendre possibles diverses formes de prolongation des études après l'école primaire et dépasser la contradiction entre égalitarisme et élitisme, démocratisation et sélection. Alors que la variété constatée des formes d'aptitudes et d'excellences conduisait les conservateurs à justifier les cloisonnements du système, elle est au contraire invoquée par les radicaux en faveur de l'ouverture à tous d'une pluralité de parcours possibles, faisant de la possibilité de ce qui sera nommé et revendiqué par la suite sous le nom de mobilité sociale non plus un péril mais une chance, tant pour l'individu que pour la société. Le caractère démocratique de ce nouveau système ne résidait cependant pas dans la promesse d'une ascension sociale pour tous, mais dans l'attribution à chacun de la place qui devait lui revenir au regard de ses aptitudes et dans la recherche des conditions politiques qui garantiraient l'égale dignité sociale de toutes les positions professionnelles. Quant à la question de savoir comment la diversité des aptitudes ainsi révélée pourrait trouver à s'employer dans les activités professionnelles disponibles, elle semble être restée dans l'ombre.

Quel est l'intérêt d'un tel détour historique, aujourd'hui ? À un moment où le principe du collège unique est interrogé, il peut être utile de revisiter une période où l'unification ne se concevait que sous la forme d'une diversification, et où le processus vers l'école unique, n'ayant pas acquis le statut d'une évidence, n'était pas encore apparu comme l'horizon indépassable d'une institution scolaire démocratique.

Frédéric Mole

frederic.mole@inrp.fr Institut national de recherche pédagogique

UMR Éducation \& politiques (INRP-université Lumière-Lyon 2)

\section{NOTES}

(1) Sur le débat entre Jaurès et Buisson au sujet de ce congrès de la Ligue de l'enseignement, je renvoie à mon étude " De l'"éducation intégrale" à la sélection des élites " (Mole, à paraître).

(2) Sur cette question, voir mon étude "De l'“éducation intégrale" à la sélection des élites » (Mole, à paraître)

(3) II faut rappeler ici que les socialistes révolutionnaires visent eux aussi une société où une "instruction généralisée " devrait permettre d'" obtenir de chacun tout ce qu'il peut produire » et de " porter la productivité humaine à son maximum ", ([Anonyme], "L'instruction du point de vue socialiste ", L'Égalité, 3 février
1878). Ferdinand Buisson ne l'ignore pas en recourant à la notion de rendement.

(4) F. Buisson et al. (1910, p. 4). Précisons que des députés socialistes sont co-signataires des projets de loi de F. Buisson : Jean Bouveri en 1910 et 1913, Léon Bétoulle et Arthur Groussier en 1913 (voir Briand \& Chapoulie, 1992, p. 395).

(5) Dans sa proposition de loi de 1913, Buisson n'introduit que quelques infimes variations, dont la suivante : " un sentiment d'égalité que la dissemblance des carrières ne saurait détruire " (Buisson et al., 1913, p. 14 ; je souligne). L'insistance dans la conviction y trahirait-elle le doute?

\section{BIBLIOGRAPHIE}

Bertrand A. (1898). L'enseignement intégral. Paris : F. Alcan.

BriAnd A. (1906). "Discours au XXVIe Congrès". Bulletin de la Ligue française de l'enseignement, octobrenovembre-décembre 1906.

Briand J.-P. \& Chapoulie J.-M. (1992). Les collèges du peuple: l'enseignement primaire supérieur et le développement de la scolarisation prolongée sous la Troisième République. Paris : INRP ; CNRS ; Fontenayaux-Roses : ENS de Fontenay-Saint-Cloud.

Buisson F. (1906a). "Désharmonie ". Le Radical, 14 août 1906.
Buisson F. (1906b). « Droits de l'enfant et devoir de l'État ". Le Petit Méridional, 8 septembre 1906.

Buisson F. (1906c). "Discours au XXVIe Congrès ». Bulletin de la Ligue française de l'enseignement, octobrenovembre-décembre 1906.

Buisson F. (1908a). La politique radicale : études sur les doctrines du parti radical et radical-socialiste. Paris : Giard \& Brière.

Buisson F. [prés.] (1908b). Le complément et l'achèvement de l'œuvre scolaire de la Troisième République: rapport de la commission de l'enseignement au $8^{\mathrm{e}}$ Congrès radical et radical-socialiste (Dijon, 1908), présenté par 
F. Buisson. Paris : Comité exécutif du Parti républicain radical \& radical-socialiste.

Buisson F. (1909). " "L'égalité du départ" 》. Manuel général de l'instruction primaire, $77^{\mathrm{e}}$ année, $8^{\mathrm{e}}$ série, t. XLVI, $\mathrm{n}^{\circ}$ 12, 4 décembre 1909.

Buisson F. (1910). "Le rôle social de l'instituteur ». Bulletin trimestriel de l'Amicale primaire et de la Ligue autonome de l'Ain, $\mathrm{n}^{\circ} 31$.

Buisson F. ; Gérard-Varet L. \& Bouveri J. (1910). Proposition de loi tendant à établir l'égalité des enfants pour le droit à l'instruction (France : Chambre des députés, $9^{\mathrm{e}}$ législature, session de 1910, proposition $n^{\circ} 3265$ ).

Buisson F. (1911). Nouveau dictionnaire de pédagogie et d'instruction primaire. Paris: Hachette. Disponible sur Internet à l'adresse: http://www.inrp.fr/editionelectronique/lodel/dictionnaire-ferdinand-buisson/ (consulté le 15 mai 2007).

Buisson F. (1912). La Foi laïque. Paris : Hachette. Document disponible au format PDF sur Internet à l'adresse : http://gallica.bnf.fr/ (consulté le 14 juin 2007)

Buisson F. ; Groussier A. ; Vincent D. ; Bétoulle L \& Bouveri J. (1913). Proposition de loi tendant à établir l'égalité des enfants pour le droit à l'instruction (France : Chambre des députés, proposition $\left.n^{\circ} 2465\right)$.

Buisson F. (1925). "L'École unique ». Revue de métaphysique et de morale, $\mathrm{n}^{\circ} 4$, p. 539-547.

Buisson F. (1933). "L'achèvement de l'œuvre scolaire de la III République ". In C. Bouglé. Un moraliste laïque, Ferdinand Buisson. Paris : F. Alcan, p. 76-100 [1 ${ }^{\text {re }}$ publication dans le Bulletin de la Ligue des droits de l'Homme, mai 1914].

Carnaud M. [prés.] (1909) Rapport sur la proposition de loi de $M$. Carnaud ayant pour but de réaliser l'« égalité des enfants devant l'instruction " par la recherche de leurs aptitudes et la coordination des divers enseignements de l'État, présenté par M. Carnaud (France: Chambre des députés, Commission de l'enseignement et des Beaux Arts, 9 e législature, session de 1909, proposition $\left.n^{\circ} 2655\right)$.

COMPAYRÉ G. (1898). “L'enseignement intégral, d'après un livre récent». Revue philosophique de la France de l'étranger, vol. 23, n 7, p. 19-44.

DeNIS D. (2006). "La dualité des ordres scolaires dans le Dictionnaire de pédagogie : entre bonne et mauvaise conscience ? ". In D. Denis \& P. Kahn (dir.), L'École de la Troisième République en questions : débats et controverses dans le Dictionnaire de pédagogie de Ferdinand Buisson. Bern : P. Lang, p. 223-255.

Fouillée A. (1898). "L'idéal socialiste de l'instruction intégrale ». Revue bleue, 5 novembre, p. 577-583.

GAVOILLE J. (1983). «L'obligation scolaire, un quart de siècle après Jules Ferry: le législateur face aux réalités ». In W. Frijhoff (dir.), L'offre d'école. éléments pour une étude comparée des politiques éducatives au XIX siècle. Paris: Publications de la Sorbonne ; INRP, p. 341-355.

GrÉARD O. (1889). Éducation et instruction. Paris : Hachette.

GuESDE J. (1899). Le socialisme au jour le jour. Paris : Giard \& Brière.

HAMELINE D. (2004). "Éducation libérale contre éducation intégrale ». In A. Ohayon, D. Ottavi \& A. Savoye (éd.), L'Éducation nouvelle, histoire, présence et devenir. Bern : P. Lang, p. 47-65.

Hirschmann A. (1991). Deux siècles de rhétorique réactionnaire. Paris : Fayard.

JAURÈs J. (1906). “Après le Congrès d'Angers 》. L'Humanité, 7 août 1906.

LANGEVIN P. \& WALLON H. [dir.] (1947). Rapport de la Commission ministérielle d'études pour la réforme de l'enseignement. Document disponible sur Internet à l'adresse : http://institut.fsu.fr/kiosque/textes_publies/ proket_langevin_wallon.htm (consulté le 11 juin 2007).

MoLE F. (à paraître). "De l'“éducation intégrale” à la sélection des élites: déplacements d'une idée pédagogique révolutionnaire dans les années 1900 ». In Actes du colloque "Les idées et les faits font-ils des histoires en éducation? »(Rouen, 18 et 19 mai 2006). Rouen; Le Havre : Publications des universités de Rouen et du Havre.

PARTI OUVRIER SOCIALISTE RÉVOLUTIONNAIRE (1897). « Rapports divers sur l'instruction intégrale". In Compte rendu du XIV congrès national du Parti ouvrier socialiste révolutionnaire, Paris: $24-25$ septembre 1896. Paris : J. Allemane.

Prost A. (1997). Éducation, société et politiques : une histoire de l'enseignement en France de 1945 à nos jours. Paris : Éd. du Seuil [Nouvelle édition augmentée].

Rıвот A. (1899). Enquête sur l'enseignement secondaire, procès-verbaux de dépositions présentés par M. Ribot, président de la commission de l'enseignement, Paris: Imprimerie des journaux officiels.

RoBIN P. (1869). «L'enseignement intégral ». La philosophie positive, septembre-octobre 1869.

SÉAILLES G. (1906). "L'égalité des enfants devant la vie et devant l'instruction: communication adressée au congrès des Jeunesses laïques de 1906 ». Manuel général de l'instruction primaire, 6 octobre 1906.

TeRral H. (2006). " Le point de vue "primaire" sur la dualité scolaire 1878-1918». In D. Denis \& P. Kahn (dir.), L'École de la Troisième République en questions: débats et controverses dans le Dictionnaire de pédagogie de Ferdinand Buisson. Bern : P. Lang, p. 209-222.

Thalamas A. (1906). "L'égalité des enfants devant l'instruction: rapport au XXV|e Congrès". Bulletin de la Ligue française de l'enseignement, octobre-novembredécembre 1906. 